Acta Crystallographica Section E

Structure Reports

Online

ISSN 1600-5368

\section{1-Aminopyridinium triiodidoplumbate(II)}

\section{Shan-Shan Yu, Hua Xian and Hai-Bao Duan*}

Department of Chemistry, Nanjing Xiaozhuang College, Nanjing 210017, People's Republic of China

Correspondence e-mail: duanhaibao4660@163.com

Received 21 August 2010; accepted 9 September 2010

Key indicators: single-crystal X-ray study; $T=296 \mathrm{~K}$; mean $\sigma(\mathrm{C}-\mathrm{C})=0.013 \AA$; disorder in main residue; $R$ factor $=0.025 ; w R$ factor $=0.056$; data-to-parameter ratio $=26.3$.

The title complex, $\left(\mathrm{C}_{5} \mathrm{H}_{7} \mathrm{~N}_{2}\right)\left[\mathrm{PbI}_{3}\right]$, consists of a 1-aminopyridinium cation, disordered about a mirror plane, and a $\left[\mathrm{PbI}_{3}\right]^{-}$anion. The $\mathrm{Pb}^{2+}$ ion ( site symmetry $\overline{1}$ ) is surrounded by six I atoms in a slightly distorted octahedral coordination. The $\mathrm{PbI}_{6}$ octahedra share faces, building up ${ }_{\infty}^{1}\left[\mathrm{PbI}_{6 / 2}\right]$ chains running along [010]. The cations are situated between the chains. Coulombic attractions and van der Waals interactions between the inorganic and organic components are mainly responsible for the cohesion of the structure.

\section{Related literature}

For background to hybrid materials, see: Rogow et al. (2010); Thirumurugan \& Rao (2008). For structures with lead halide building blocks, see: Li et al. (2008); Zhang et al. (2008).

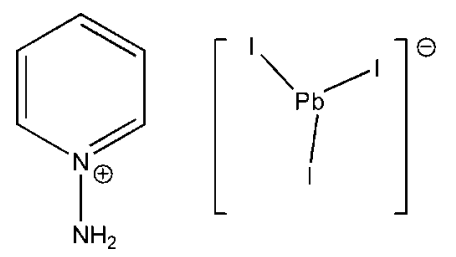

\section{Experimental}

Crystal data

$\left(\mathrm{C}_{5} \mathrm{H}_{7} \mathrm{~N}_{2}\right)\left[\mathrm{PbI}_{3}\right]$

$M_{r}=683.03$

Orthorhombic, Pnma

$a=15.0417$ (19) $\AA$

$b=8.1316(10) \AA$

$c=10.5625(14) \AA$

$$
\begin{aligned}
& V=1291.9(3) \AA^{3} \\
& Z=4 \\
& \text { Mo } K \alpha \text { radiation } \\
& \mu=20.18 \mathrm{~mm}^{-1} \\
& T=296 \mathrm{~K} \\
& 0.6 \times 0.2 \times 0.1 \mathrm{~mm}
\end{aligned}
$$

Data collection

Bruker SMART CCD area-detector diffractometer

Absorption correction: multi-scan

(SADABS; Bruker, 2000)

$T_{\min }=0.011, T_{\max }=0.133$

10792 measured reflections 1607 independent reflections 1263 reflections with $I>2 \sigma(I)$

$R_{\text {int }}=0.036$

Refinement

$R\left[F^{2}>2 \sigma\left(F^{2}\right)\right]=0.025$

$w R\left(F^{2}\right)=0.056$

$S=1.11$

1607 reflections

61 parameters

1 restraint

$\mathrm{H}$-atom parameters constrained

$\Delta \rho_{\max }=0.86 \mathrm{e}^{-3}$

$\Delta \rho_{\min }=-1.05$ e $\AA^{-3}$

Table 1

Selected bond lengths ( $\mathrm{A})$.

\begin{tabular}{llll}
\hline $\mathrm{Pb} 1-\mathrm{I} 3$ & $3.2301(5)$ & $\mathrm{Pb} 1-\mathrm{I} 2$ & $3.2412(5)$ \\
$\mathrm{Pb} 1-\mathrm{I} 1$ & $3.2303(5)$ & & \\
\hline
\end{tabular}

Data collection: SMART (Bruker, 2000); cell refinement: SAINT (Bruker, 2000); data reduction: $S A I N T$; $\operatorname{program}(\mathrm{s})$ used to solve structure: SHELXTL (Sheldrick, 2008); program(s) used to refine structure: SHELXTL; molecular graphics: SHELXTL; software used to prepare material for publication: $S H E L X T L$.

The authors thank the Science and Technology Department of Jiangsu Province for financial support (grant No 10774076).

Supplementary data and figures for this paper are available from the IUCr electronic archives (Reference: WM2395).

\title{
References
}

Bruker (2000). SMART, SAINT and SADABS. Bruker AXS Inc., Madison, Wisconsin, USA.

Li, Y., Zheng, G., Lin, C. \& Lin, J. (2008). Cryst. Growth Des. 8, 1990-1996.

Rogow, D. L., Russell, M. P., Wayman, L. M., Swanson, C. H., Oliver, A. G. \& Oliver, S. R. J. (2010). Cryst. Growth Des. 10, 823-829.

Sheldrick, G. M. (2008). Acta Cryst. A64, 112-122.

Thirumurugan, A. \& Rao, C. N. R. (2008). Cryst. Growth Des. 8, 1640-1644.

Zhang, Z. J., Xiang, S. C. \& Guo, G. C. (2008). Angew. Chem. Int. Ed. 47, 41494152. 


\section{supporting information}

Acta Cryst. (2010). E66, m1259［doi:10.1107/S1600536810036263]

\section{1-Aminopyridinium triiodidoplumbate(II)}

\section{Shan-Shan Yu, Hua Xian and Hai-Bao Duan}

\section{S1. Comment}

Inorganic metal-halide building-blocks have received special attention with respect to the construction of inorganicorganic hybrid materials (Rogow et al., 2010; Thirumurugan et al., 2008). Among these materials octahedral building blocks of lead halides are frequently found and numerous crystal structures, from one-dimensional chains to threedimensional frameworks (Li et al., 2008; Zhang et al., 2008), were observed. Herein we report the crystal structure of the title compound, $\left(\mathrm{C}_{5} \mathrm{H}_{7} \mathrm{~N}_{2}\right)\left[\mathrm{PbI}_{3}\right]$ (I).

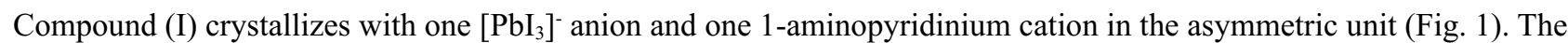
cation is disordered about a mirror plane. The $\mathrm{Pb}^{2+}$ cation is coordinated by six iodide anions in a slightly distorted octahedral coordination geometry. The $\mathrm{PbI}_{6}$ octahedra share faces, resulting in anionic chains running along [010]. As show in Fig. 2., the straight inorganic chains are embedded in cationic stacks. Besides Coulomb attractions, only weak van der Waals interactions between the inorganic and organic components exist.

\section{S2. Experimental}

A mixture of $\mathrm{PbI}_{2}(922 \mathrm{mg}, 2.0 \mathrm{mmol})$ and 1-aminopyridinium iodide $(190 \mathrm{mg}, 2.0 \mathrm{mmol})$ in a molar ratio of 1:1 in DMF was slowly evaporated to produce orange needle-shaped crystals.

\section{S3. Refinement}

The $\mathrm{H}$ atoms were placed in geometrically idealized positions and refined as riding atoms, with $U_{\mathrm{iso}}(\mathrm{H})=1.2 U_{\mathrm{eq}}(\mathrm{C})$. The cation is disordered about a mirror plane. Atoms $\mathrm{C} 3$ and $\mathrm{N} 1$ occupy the same site with an occupation factor of $50 \%$. The respective $-\mathrm{NH}_{2}$ group and the $\mathrm{H}$ atom show likewise half-occupation. 


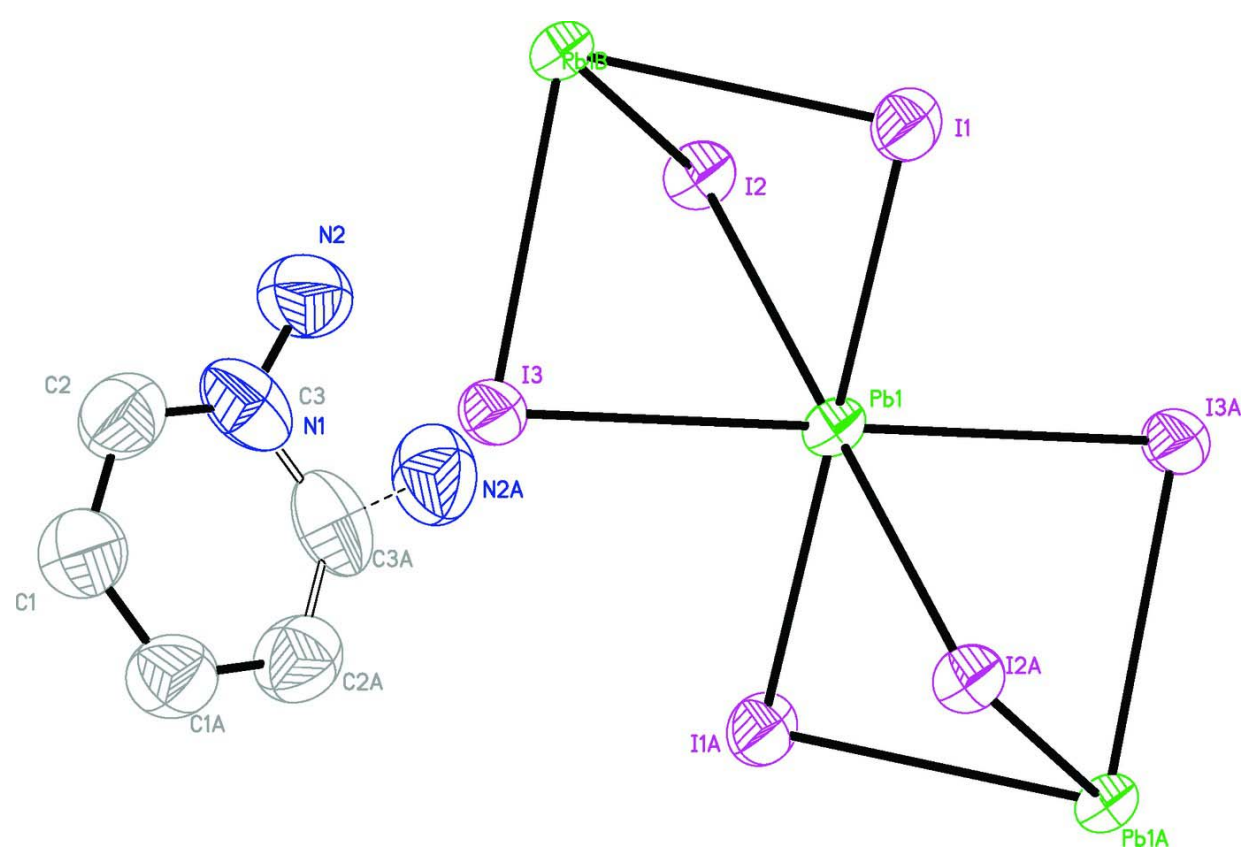

\section{Figure 1}

Part of the structure of (I), showing the atom-numbering scheme and displacement ellipsoids at the $30 \%$ probability level. Atom $\mathrm{C} 3$ and $\mathrm{N} 1$ are positionally disordered. $\mathrm{H}$ atoms have been omitted for clyrity. [Symmetry code: $\mathrm{A} x, 0.5-y, z$.] 


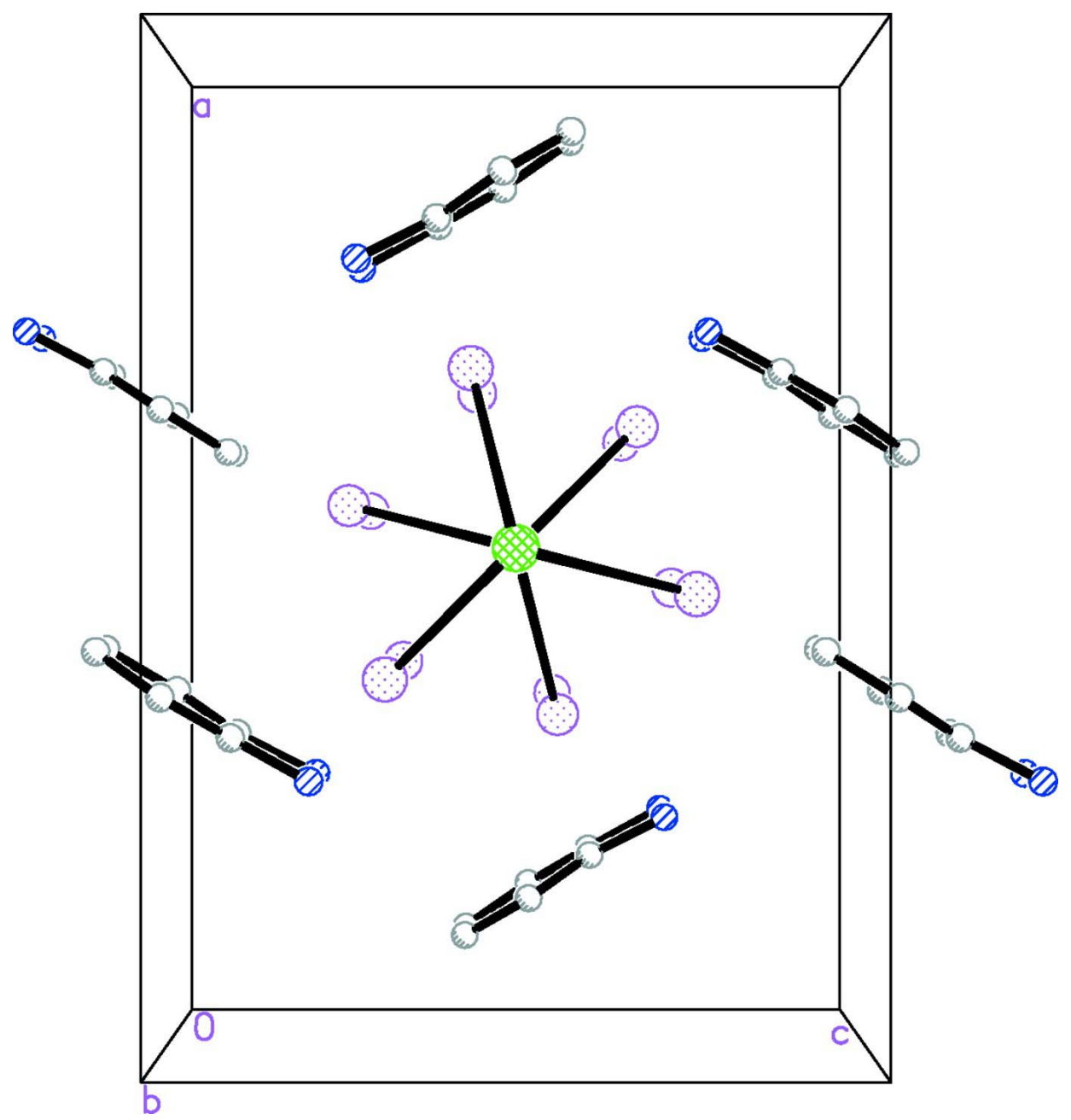

\section{Figure 2}

The alignment of inorganic and organic components in the crystals of 1 along [010].

\section{1-Aminopyridinium triiodidoplumbate(II)}

Crystal data

$\left(\mathrm{C}_{5} \mathrm{H}_{7} \mathrm{~N}_{2}\right)\left[\mathrm{PbI}_{3}\right]$

$M_{r}=683.03$

Orthorhombic, Pnma

Hall symbol: -P 2ac 2n

$a=15.0417$ (19) $\AA$

$b=8.1316(10) \AA$

$c=10.5625(14) \AA$

$V=1291.9(3) \AA^{3}$

\section{Data collection}

Bruker SMART CCD area-detector diffractometer

Radiation source: fine-focus sealed tube Graphite monochromator phi and $\omega$ scans
$Z=4$

$F(000)=1168$

$D_{\mathrm{x}}=3.512 \mathrm{Mg} \mathrm{m}^{-3}$

Mo $K \alpha$ radiation, $\lambda=0.71073 \AA$

$\mu=20.18 \mathrm{~mm}^{-1}$

$T=296 \mathrm{~K}$

Needle, orange

$0.6 \times 0.2 \times 0.1 \mathrm{~mm}$

Absorption correction: multi-scan

$$
\text { (SADABS; Bruker, 2000) }
$$

$T_{\min }=0.011, T_{\max }=0.133$

10792 measured reflections

1607 independent reflections

1263 reflections with $I>2 \sigma(I)$ 
$R_{\text {int }}=0.036$

$\theta_{\max }=27.6^{\circ}, \theta_{\min }=2.4^{\circ}$

$h=-19 \rightarrow 19$

\section{Refinement}

Refinement on $F^{2}$

Least-squares matrix: full

$R\left[F^{2}>2 \sigma\left(F^{2}\right)\right]=0.025$

$w R\left(F^{2}\right)=0.056$

$S=1.11$

1607 reflections

61 parameters

1 restraint

Primary atom site location: structure-invariant direct methods $k=-10 \rightarrow 10$

$l=-13 \rightarrow 13$

Secondary atom site location: difference Fourier map

Hydrogen site location: inferred from neighbouring sites

$\mathrm{H}$-atom parameters constrained

$w=1 /\left[\sigma^{2}\left(F_{\mathrm{o}}^{2}\right)+(0.016 P)^{2}+2.8133 P\right]$ where $P=\left(F_{\mathrm{o}}^{2}+2 F_{\mathrm{c}}^{2}\right) / 3$

$(\Delta / \sigma)_{\max }=0.001$

$\Delta \rho_{\max }=0.86 \mathrm{e}^{-3}$

$\Delta \rho_{\min }=-1.05$ e $\AA^{-3}$

Special details

Geometry. All e.s.d.'s (except the e.s.d. in the dihedral angle between two 1.s. planes) are estimated using the full covariance matrix. The cell e.s.d.'s are taken into account individually in the estimation of e.s.d.'s in distances, angles and torsion angles; correlations between e.s.d.'s in cell parameters are only used when they are defined by crystal symmetry. An approximate (isotropic) treatment of cell e.s.d.'s is used for estimating e.s.d.'s involving 1.s. planes.

Refinement. Refinement of $F^{2}$ against ALL reflections. The weighted $R$-factor $w R$ and goodness of fit $S$ are based on $F^{2}$, conventional $R$-factors $R$ are based on $F$, with $F$ set to zero for negative $F^{2}$. The threshold expression of $F^{2}>\sigma\left(F^{2}\right)$ is used only for calculating $R$-factors(gt) etc. and is not relevant to the choice of reflections for refinement. $R$-factors based on $F^{2}$ are statistically about twice as large as those based on $F$, and $R$ - factors based on ALL data will be even larger.

Fractional atomic coordinates and isotropic or equivalent isotropic displacement parameters $\left(\AA^{2}\right)$

\begin{tabular}{llllll}
\hline & $x$ & $y$ & $z$ & $U_{\text {iso }} * / U_{\text {eq }}$ & Occ. $(<1)$ \\
\hline $\mathrm{Pb} 1$ & 0.5000 & 0.5000 & 0.5000 & $0.05028(11)$ & \\
$\mathrm{I} 1$ & $0.66188(4)$ & 0.2500 & $0.44218(7)$ & $0.06995(19)$ & \\
$\mathrm{I} 2$ & $0.45861(4)$ & 0.2500 & $0.73162(5)$ & $0.05961(16)$ & \\
$\mathrm{I} 3$ & $0.38187(5)$ & 0.2500 & $0.33215(6)$ & $0.06837(19)$ & \\
$\mathrm{C} 1$ & $0.0996(6)$ & $0.1682(10)$ & $0.4247(8)$ & $0.100(3)$ & \\
$\mathrm{H} 1$ & 0.0708 & 0.1100 & 0.3609 & $0.120^{*}$ & \\
$\mathrm{C} 2$ & $0.1419(7)$ & $0.0883(12)$ & $0.5186(9)$ & $0.103(3)$ & \\
$\mathrm{H} 2$ & 0.1424 & -0.0261 & 0.5197 & $0.123^{*}$ & 0.50 \\
$\mathrm{C} 3$ & $0.1824(5)$ & $0.1708(11)$ & $0.6087(7)$ & $0.100(3)$ & 0.50 \\
$\mathrm{H} 3$ & 0.2114 & 0.1142 & 0.6731 & $0.120^{*}$ & 0.50 \\
$\mathrm{~N} 1$ & $0.1824(5)$ & $0.1708(11)$ & $0.6087(7)$ & $0.100(3)$ & 0.50 \\
$\mathrm{~N} 2$ & $0.2230(10)$ & $0.132(2)$ & $0.7177(16)$ & $0.121(5)$ & 0.50 \\
$\mathrm{H} 2 \mathrm{~A}$ & 0.2415 & 0.2091 & 0.7672 & $0.146^{*}$ & 0.50 \\
$\mathrm{H} 2 \mathrm{~B}$ & 0.2307 & 0.0310 & 0.7382 & $0.146^{*}$ & \\
\end{tabular}

Atomic displacement parameters $\left(\AA^{2}\right)$

\begin{tabular}{lllllll}
\hline & $U^{11}$ & $U^{22}$ & $U^{33}$ & $U^{12}$ & $U^{13}$ & $U^{23}$ \\
\hline Pb1 & $0.0585(2)$ & $0.03787(16)$ & $0.05448(19)$ & $0.00017(13)$ & $0.00130(15)$ & $-0.00097(14)$ \\
I1 & $0.0549(3)$ & $0.0611(4)$ & $0.0938(5)$ & 0.000 & $0.0189(3)$ & 0.000 \\
I2 & $0.0727(4)$ & $0.0575(3)$ & $0.0486(3)$ & 0.000 & $0.0023(3)$ & 0.000 \\
I3 & $0.0753(4)$ & $0.0603(4)$ & $0.0696(4)$ & 0.000 & $-0.0268(3)$ & 0.000
\end{tabular}




$\begin{array}{lllllll}\mathrm{C} 1 & 0.120(7) & 0.100(6) & 0.081(5) & -0.001(5) & -0.015(5) & -0.010(5) \\ \mathrm{C} 2 & 0.104(7) & 0.089(6) & 0.114(8) & -0.005(5) & 0.008(6) & 0.004(6) \\ \mathrm{C} 3 & 0.064(4) & 0.162(9) & 0.074(5) & 0.010(4) & 0.010(3) & 0.026(5) \\ \mathrm{N} 1 & 0.064(4) & 0.162(9) & 0.074(5) & 0.010(4) & 0.010(3) & 0.026(5) \\ \mathrm{N} 2 & 0.111(11) & 0.121(12) & 0.133(13) & 0.004(10) & -0.004(11) & 0.029(11)\end{array}$

Geometric parameters $\left(\stackrel{A}{\circ}{ }^{\circ}\right)$

\begin{tabular}{|c|c|c|c|}
\hline $\mathrm{Pb} 1-\mathrm{I}^{\mathrm{i}}$ & $3.2301(5)$ & $\mathrm{C} 1-\mathrm{C}^{\mathrm{iii}}$ & $1.331(16)$ \\
\hline $\mathrm{Pb} 1-\mathrm{I} 3$ & $3.2301(5)$ & $\mathrm{C} 1-\mathrm{C} 2$ & $1.345(12)$ \\
\hline $\mathrm{Pb} 1-\mathrm{I} 1$ & $3.2303(5)$ & $\mathrm{C} 1-\mathrm{H} 1$ & 0.9300 \\
\hline $\mathrm{Pb} 1-\mathrm{I} 1^{\mathrm{i}}$ & $3.2303(5)$ & $\mathrm{C} 2-\mathrm{C} 3$ & $1.315(11)$ \\
\hline $\mathrm{Pb} 1-\mathrm{I} 2$ & $3.2412(5)$ & $\mathrm{C} 2-\mathrm{H} 2$ & 0.9300 \\
\hline $\mathrm{Pb} 1-\mathrm{I} 2^{\mathrm{i}}$ & $3.2412(5)$ & $\mathrm{C} 3-\mathrm{C} 3^{\mathrm{iii}}$ & $1.288(18)$ \\
\hline $\mathrm{I} 1-\mathrm{Pb} 1^{\mathrm{ii}}$ & $3.2303(5)$ & $\mathrm{C} 3-\mathrm{H} 3$ & 0.9300 \\
\hline $\mathrm{I} 2-\mathrm{Pb} 1^{\mathrm{ii}}$ & $3.2412(5)$ & $\mathrm{N} 2-\mathrm{H} 2 \mathrm{~A}$ & 0.8600 \\
\hline $\mathrm{I} 3-\mathrm{Pb} 1^{\mathrm{ii}}$ & $3.2301(5)$ & $\mathrm{N} 2-\mathrm{H} 2 \mathrm{~B}$ & 0.8600 \\
\hline $\mathrm{I} 3^{\mathrm{i}}-\mathrm{Pb} 1-\mathrm{I} 3$ & 180.0 & $\mathrm{I} 2-\mathrm{Pb} 1-\mathrm{I} 2^{\mathrm{i}}$ & 180.0 \\
\hline 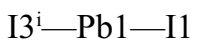 & $94.886(17)$ & $\mathrm{Pb} 1 \mathrm{ii}-\mathrm{I} 1-\mathrm{Pb} 1$ & $78.000(17)$ \\
\hline $\mathrm{I} 3-\mathrm{Pb} 1-\mathrm{I} 1$ & $85.114(17)$ & $\mathrm{Pb} 1^{\mathrm{ii}}-\mathrm{I} 2-\mathrm{Pb} 1$ & $77.688(16)$ \\
\hline $\mathrm{I} 3^{\mathrm{i}}-\mathrm{Pb} 1-\mathrm{I} 1^{\mathrm{i}}$ & $85.114(17)$ & $\mathrm{Pb} 1-\mathrm{I} 3-\mathrm{Pb} 1^{\mathrm{ii}}$ & $78.007(16)$ \\
\hline $\mathrm{I} 3-\mathrm{Pb} 1-\mathrm{I} 1^{\mathrm{i}}$ & $94.886(17)$ & $\mathrm{C} 1{ }^{\mathrm{iii}}-\mathrm{C} 1-\mathrm{C} 2$ & $118.9(6)$ \\
\hline $\mathrm{I} 1-\mathrm{Pb} 1-\mathrm{I} 1^{\mathrm{i}}$ & 180.0 & 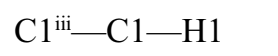 & 120.6 \\
\hline $\mathrm{I} 3^{\mathrm{i}}-\mathrm{Pb} 1-\mathrm{I} 2$ & $94.940(16)$ & $\mathrm{C} 2-\mathrm{C} 1-\mathrm{H} 1$ & 120.6 \\
\hline $\mathrm{I} 3-\mathrm{Pb} 1-\mathrm{I} 2$ & $85.060(16)$ & $\mathrm{C} 3-\mathrm{C} 2-\mathrm{C} 1$ & $120.4(9)$ \\
\hline $\mathrm{I} 1-\mathrm{Pb} 1-\mathrm{I} 2$ & $83.844(15)$ & $\mathrm{C} 3-\mathrm{C} 2-\mathrm{H} 2$ & 119.8 \\
\hline $\mathrm{I} 1{ }^{\mathrm{i}}-\mathrm{Pb} 1-\mathrm{I} 2$ & $96.156(15)$ & $\mathrm{C} 1-\mathrm{C} 2-\mathrm{H} 2$ & 119.8 \\
\hline $\mathrm{I} 3^{\mathrm{i}}-\mathrm{Pb} 1-\mathrm{I} 2^{\mathrm{i}}$ & $85.060(16)$ & $\mathrm{C} 3^{\mathrm{iii}-\mathrm{C} 3-\mathrm{C} 2}$ & $120.7(6)$ \\
\hline $\mathrm{I} 3-\mathrm{Pb} 1-\mathrm{I} 2^{\mathrm{i}}$ & $94.940(16)$ & $\mathrm{C} 3^{\mathrm{iii}-\mathrm{C} 3-\mathrm{H} 3}$ & 119.7 \\
\hline $\mathrm{I} 1-\mathrm{Pb} 1-\mathrm{I} 2^{\mathrm{i}}$ & $96.156(15)$ & $\mathrm{C} 2-\mathrm{C} 3-\mathrm{H} 3$ & 119.7 \\
\hline $\mathrm{I} 1^{\mathrm{i}}-\mathrm{Pb} 1-\mathrm{I} 2^{\mathrm{i}}$ & $83.844(15)$ & $\mathrm{H} 2 \mathrm{~A}-\mathrm{N} 2-\mathrm{H} 2 \mathrm{~B}$ & 120.0 \\
\hline
\end{tabular}

Symmetry codes: (i) $-x+1,-y+1,-z+1$; (ii) $-x+1, y-1 / 2,-z+1$; (iii) $x,-y+1 / 2, z$. 